\title{
An Application of the Adomian Decomposition Method for Inverse Stefan Problem with Neumann's Boundary Condition
}

\author{
Radosław Grzymkowski and Damian Słota \\ Institute of Mathematics, Silesian University of Technology, Kaszubska 23, \\ 44-100 Gliwice, Poland \\ $\{$ r.grzymkowski, d.slota\}@polsl.pl
}

\begin{abstract}
In this paper the solution of one-phase inverse Stefan problem with Neumann's boundary condition is presented. This problem consists of the reconstruction of the function which describes the heat flux on the boundary, when the position of the moving interface is well-known. The proposed solution is based on the Adomian decomposition method and the least square method.
\end{abstract}

\section{Introduction}

In this paper we solve the one-phase inverse Stefan problem, which consists of the reconstruction of the function which describes the heat flux on the boundary, when the position of the moving interfaces is well-known. This kind of problem becomes an inverse design problem. The conditions for the existence and uniqueness of the solution of this problem are given in the literature [3. The solution is based on the Adomian decomposition method and the least square method.

The Adomian decomposition method was developed by G. Adomian [1,2]. This method is useful for solving a wide class of problems 2, 4. Using this method we are able to solve non-linear operator equation:

$$
F(u)=f
$$

where $F: H \rightarrow G$ is a non-linear operator, $f$ is a known element from Hilbert space $G$ and $u$ is the sought element from Hilbert space $H$. Operator $F(u)$ can be written as:

$$
F(u)=L(u)+R(u)+N(u),
$$

where $L$ is the invertible linear operator, $R$ is the remaining linear operator and $N$ is a non-linear operator. The solution of the equation (11) is sought in the form of a functional series:

$$
u=\sum_{i=0}^{\infty} g_{i} .
$$

After some manipulations we obtain the following recurrent formula:

$$
\begin{aligned}
& g_{0}=g^{*}+L^{-1}(f), \\
& g_{n}=-L^{-1} R\left(g_{n-1}\right)-L^{-1}\left(A_{n-1}\right), \quad n \geq 1,
\end{aligned}
$$


where $g^{*}$ is a function dependent on the initial and boundary conditions and $L^{-1}$ is the inverse operator.

\section{Inverse Stefan Problem}

Let $D \subset \mathbb{R}^{2}$ be a domain (Figure 1). We seek an approximate solution of the following problem:

For the given position of freezing front $\Gamma_{g}$, the distribution of temperature $u$ in domain $D$ is calculated as well as function $q(t)$ on boundary $\Gamma_{1}$, which satisfies the following equations:

$$
\begin{aligned}
\frac{\partial^{2} u(x, t)}{\partial x^{2}} & =\frac{1}{a} \frac{\partial u}{\partial t}(x, t), & & \text { in } D, \\
u(x, 0) & =\varphi(x), & & \text { on } \Gamma_{0}, \\
-\lambda \frac{\partial u(x, t)}{\partial x} & =q(t), & & \text { on } \Gamma_{1}, \\
u(\xi(t), t) & =u^{*}, & & \text { on } \Gamma_{g}, \\
-\lambda \frac{\partial u(x, t)}{\partial x} & =\kappa \frac{d \xi(t)}{d t}, & & \text { on } \Gamma_{g},
\end{aligned}
$$

where $a$ is the thermal diffusivity, $\lambda$ is the thermal conductivity, $\kappa$ is the latent heat of fusion per unit volume, and $u, t$ and $x$ refer to temperature, time and spatial location, respectively.

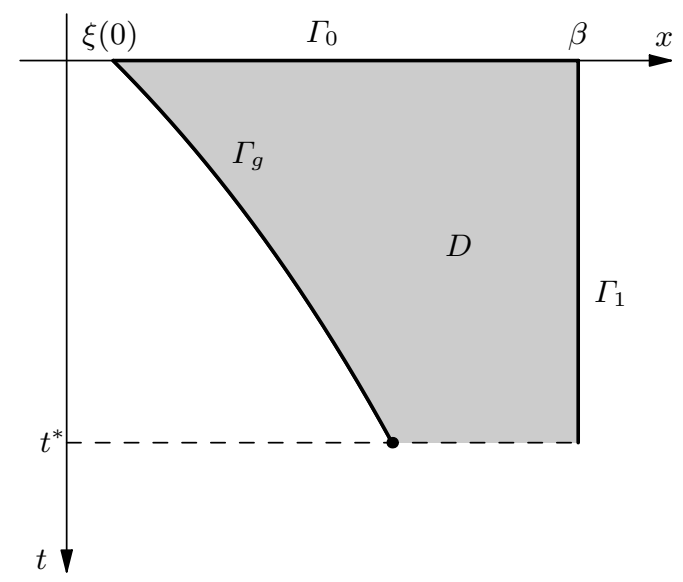

Fig. 1. Domain formulation of the problem 


\section{Solution of the Problem}

In the problem under consideration we have operator equations (1), where:

$$
L(u)=\frac{\partial^{2} u}{\partial x^{2}}, \quad R(u)=-\frac{1}{a} \frac{\partial u}{\partial t}, \quad N(u)=0, \quad f=0 .
$$

The inverse operator $L^{-1}$ is given by:

$$
L^{-1}(u)=\int_{\xi(t)}^{x} \int_{x}^{\beta} u(x, t) d x d x .
$$

Hence, using the boundary condition (7) and (8) we obtain:

$$
\begin{aligned}
& g_{0}(x, t)=\frac{1}{\lambda} q(t)(\xi(t)-x)+u^{*}, \\
& g_{n}(x, t)=-\frac{1}{a} \int_{\xi(t)}^{x} \int_{x}^{\beta} \frac{\partial g_{n-1}(x, t)}{\partial t} d x d x, \quad n \geq 1 .
\end{aligned}
$$

We seek an approximation solution in the form:

$$
u_{n}(x, t)=\sum_{i=0}^{n} g_{i}(x, t), \quad n \in \mathbb{N} .
$$

Because the $g_{i}$ (11) are dependent on the unknown function $q(t)$, we derived this function in the form:

$$
q(t)=\sum_{i=1}^{m} p_{i} \psi_{i}(t)
$$

where $p_{i} \in \mathbb{R}$ and the basis functions $\psi_{i}(t)$ are linearly independent. The coefficients $p_{i}$ are selected to show minimal deviation of function (12) from the conditions (6) and (9) (considering the assumed measure). To construct the measure of error the least square method is applied. Thus we seek the minimum of the following functional:

$$
\begin{aligned}
J\left(p_{1}, \ldots, p_{m}\right)= & \int_{\xi(0)}^{\beta}\left(u_{n}(x, 0)-\varphi(x)\right)^{2} d x+ \\
& +\int_{0}^{t^{*}}\left(\left.\lambda \frac{\partial u_{n}(x, t)}{\partial x}\right|_{x=\xi(t)}+\kappa \frac{d \xi(t)}{d t}\right)^{2} d t .
\end{aligned}
$$

Substituting equations (12), (11) and (13) to functional $J$ and differentiating it with respect to the coefficients $p_{i}(i=1, \ldots, m)$ and equating the obtained derivatives to zero, the system of the linear algebraic equations is obtained. In the course of solving this system, coefficients $p_{i}$ are determined, and thereby the approximated distributions of temperature in the domain $D$ and the heat flux on the boundary $\Gamma_{1}$ are obtained. 


\section{$4 \quad$ Numerical Example}

The theoretical considerations introduced in the previous sections will be illustrated with an example, in which: $\beta=1, a=1, \lambda=2, \xi(t)=a t, \varphi(x)=e^{-x}$, $\kappa=\lambda / a, u^{*}=1, t^{*}=1$. Then the exact solution of the inverse Stefan problem can be found from the following functions: $u(x, t)=e^{a t-x}$ for $(x, t) \in D$, and $q(t)=\lambda e^{a t-\beta}$ for $t \in\left[0, t^{*}\right]$. As basis functions we take $\psi_{i}(t)=t^{i-1}, i=$ $1, \ldots, m$. For the calculations, we assume $m \in\{2,3, \ldots, 6\}$ and $n \in\{2,3, \ldots, 7\}$.

Figure 2 shows the exact and the reconstructed heat flux on the boundary $\Gamma_{1}$ for a different number of elements of the sum (12), $n \in\{4,7\}$ and for six basis functions $\psi_{i}(t)$. The results obtained show that functions $q(t)$ and $u(x, t)$ are reconstructed very well.
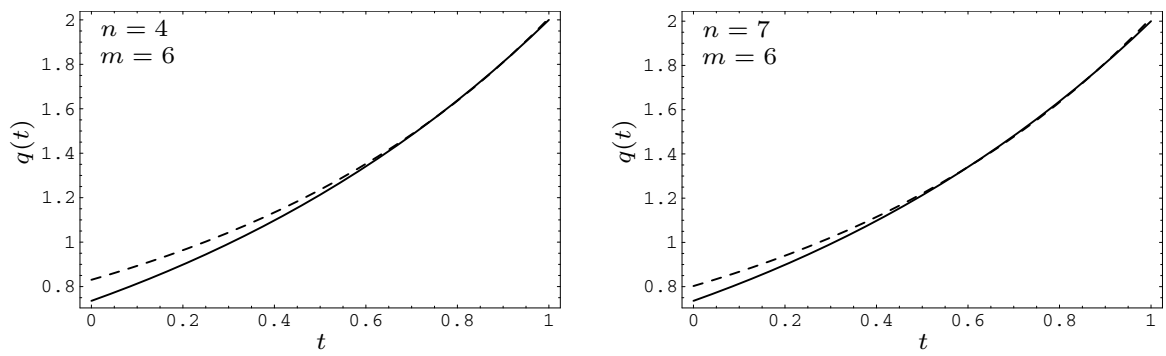

Fig. 2. The heat flux on the boundary $\Gamma_{1}$ (solid line - exact value, dash line - reconstructed value)

\section{Conclusion}

We have presented a method for solving the one-phase inverse Stefan problem which consists of the reconstruction of the function describing the heat flux on the boundary, when the position of the moving interface is well-known. The method makes use of the Adomian decomposition method and the least square method. The calculations presented show that this method is effective for solving this problem.

\section{References}

1. Adomian, G.: Stochastic Systems. Academic Press, New York (1983)

2. Adomian, G.: A review of the decomposition method in applied mathematics. J. Math. Anal. Appl. 135 (1988) 501-544

3. Goldman, N.L.: Inverse Stefan problem. Kluwer, Dordrecht (1997)

4. Lesnic, D.: Convergence of Adomian's decomposition method: periodic temperatures. Computers Math. Applic. 44 (2002) 13-24 